\title{
November 2014 Pulmonary Case of the Month: BAL Eosinophilia
}

\author{
Salma Imran Patel, MD, MPH \\ Lewis J. Wesselius, MD \\ Laszlo T. Vaszar, MD \\ Departments of Internal Medicine and Pulmonary Medicine \\ Mayo Clinic Arizona \\ Scottsdale, AZ
}

\section{History of Present IIIness}

A 62 year-old- was admitted to the hospital for 2 weeks of worsening cough, yellowish sputum production, shortness of breath and pleuritic chest pain. The patient has had asthma since the 1970s and presently uses salmeterol/fluticasone and albuterol as a rescue inhaler. He was intubated once four years ago, and has had a total of three hospitalizations for his asthma and 15 courses of prednisone. He is sensitive to cold/hot air, all animals, aspirin and acetaminophen.

\section{$\mathrm{PMH}, \mathrm{FH}, \mathrm{SH}$}

In addition to the asthma, he has a history of type 2 diabetes mellitus, hypertension, gastroesophageal reflux disease, and chronic abdominal pain.

\section{Physical Examination}

Vital signs: T $36.6^{\circ} \mathrm{C}, \mathrm{HR}$ 98, BP129/69, RR 20 and SPO2 96\% on $2 \mathrm{~L}$ of oxygen by nasal cannula. He was mildly distressed and coughing. His pulmonary exam showed diffuse inspiratory and expiratory wheezes. The remainder of his exam was unremarkable.

\section{Laboratory}

Significant findings on laboratory evaluation include an elevated white blood cell count of 13,400 cells/yL, an elevated absolute eosinophil count of 2,820 eosinophils/yL, an elevated glucose of $131 \mathrm{mg} / \mathrm{dL}$, and a low sodium of $120 \mathrm{mEq} / \mathrm{L}$.

\section{Imaging}

A thoracic CT scan was performed (Figure 1). 

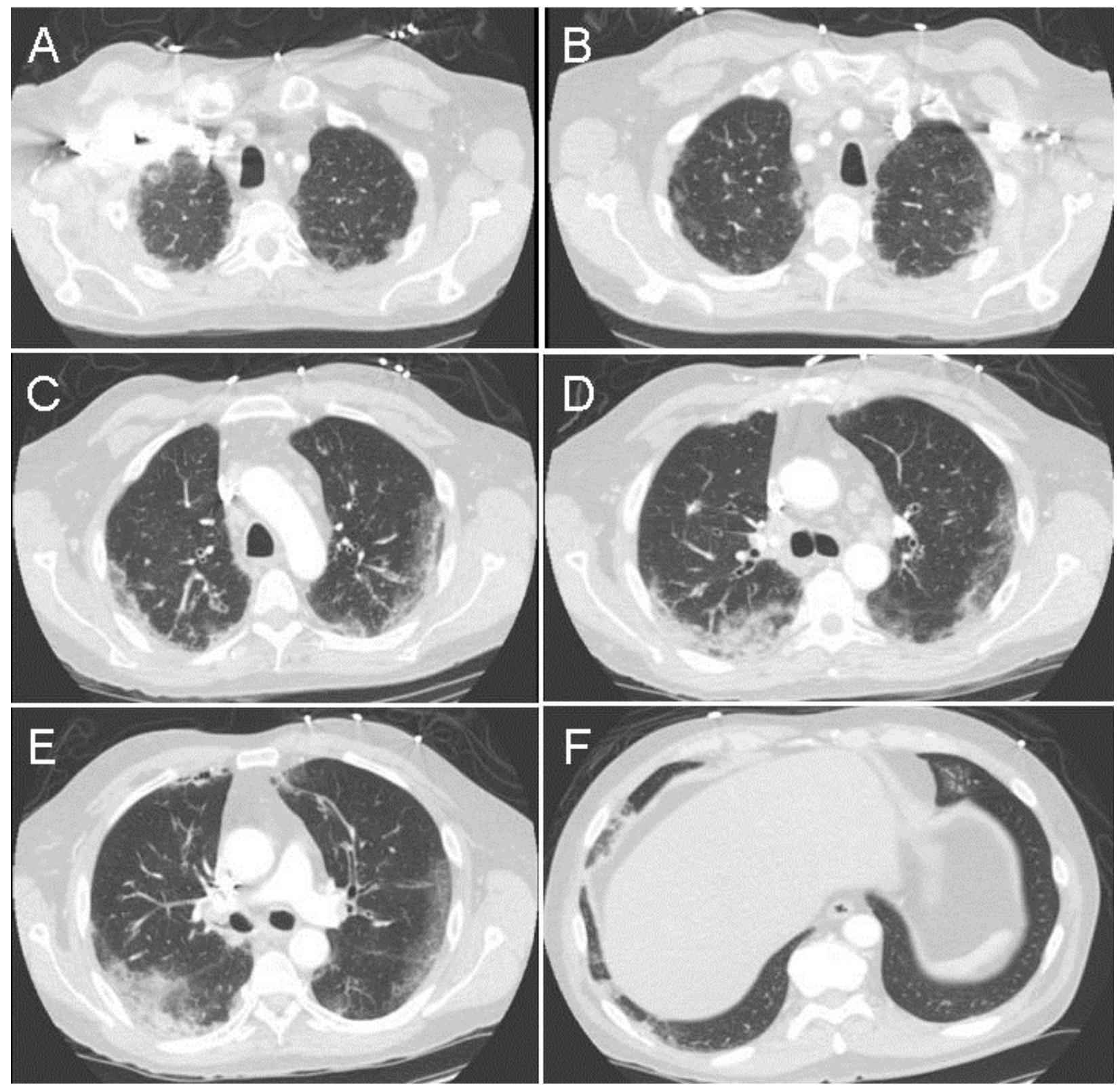

Figure 1. Representative images in lung windows from thoracic CT scan.

Which of the following best describes the CT scan?

1. Cavitary changes in both apices

2. Central consolidation

3. Fibrotic changes at the bases

4. Peripheral opacities

5. Normal 


\section{Correct! \\ 4. Peripheral opacities}

The CT scan shows opacities predominately at the periphery of the lung with relative sparing centrally. This is sometimes called a reverse bat's wing pattern of pulmonary consolidation (1).

Which of the following disorders typically have peripheral pulmonary opacities?

1. Bronchoalveolar carcinoma

2. Chronic eosinophilic pneumonia

3. Cryptogenic organizing pneumonia, formerly bronchiolitis obliterans with organizing pneumonia (BOOP)

4. Pulmonary vasculitis

5. All of the above 


\section{Correct! \\ 5. All of the above}

All are causes of reverse bat's wing opacities. Other additional causes include bronchoalveolar carcinoma, pulmonary contusion (in the setting of trauma), pulmonary infarction, and aspiration (1).

A TB-QuantiFERON, urine antigen for legionella, sputum culture, cocci serology, and anti-nuclear antibody (ANCA) were all obtained and were negative

A bronchoscopy with bronchoalveolar lavage (BAL) was performed. The BAL differential was:

Eosinophils $\quad 52 \%$

Lymphocytes $\quad 5 \%$

Alveolar macrophages $\quad 28 \%$

Neutrophils $\quad 15 \%$

Which of the following disorders is/are most likely based on the BAL eosinophil percentage?

1. Chronic eosinophilic pneumonia

2. Drug-induced pneumonitis

3. Churg-Strauss syndrome

4. 1 and 3

5. All of the above 


\section{Correct!}

\section{1 and 3}

BAL eosinophilia can be divided into those diseases associated with a high eosinophil percentage ( $\geq 25 \%)$ and those with mild to moderate elevation (<25\%) (2). Those diseases most commonly associated with a high eosinophil count include:

1. Chronic eosinophilic pneumonia (>40\%)

2. Churg Strauss syndrome

3. Acute eosinophilic pneumonia $(>25 \%)$

4. Tropical pulmonary eosinophilia $(40-70 \%)$.

Those diseases associated with mild to moderate counts include:

1. Connective tissue disease

2. Drug-induced pneumonitis

3. Fungal pneumonia

4. Idiopathic pulmonary fibrosis $(<10 \%)$

5. Pulmonary Langerhans cell histiocytosis (Histiocytosis $\mathrm{X}$ )

6. Sarcoidosis.

A clinical diagnosis of chronic eosinophilic pneumonia (CEP) was made in our patient.

Which of the following is true regarding treatment of CEP?

1. Relapses are uncommon

2. Standard treatment is with inhaled corticosteroids

3. Standard treatment is with oral corticosteroids

4. Treatment usually requires at least 3 months with high doses of corticosteroids (>30 mg of prednisone or its equivalent per day)

5. None are true 


\section{Correct! \\ 3. Standard treatment is with oral corticosteroids}

The standard treatment of CEP is with oral corticosteroids, although spontaneous remission may occur in $10 \%$ of patients (3). CEP usually responds rapidly and dramatically to corticosteroids. Failure to respond to corticosteroids suggest the diagnosis of CEP may be wrong. Inhaled steroid treatment in CEP has not been studied prospectively but one brief report of the use of inhaled steroids alone in CEP suggests it may be ineffective.

Relapses occur in up to $83 \%$ of patients (3). Symptoms and radiologic opacities often reappear when the corticosteroid dose is lowered or stopped, and patients usually require a maintenance dose equivalent to or greater than $15 \mathrm{mg}$ of prednisone per day to remain symptom free and without radiographic opacities. Addition of inhaled steroids to the oral corticosteroid regimen may allow a reduction in the oral corticosteroid maintenance dose.

Our patient was treated with an initial dose of $125 \mathrm{mg}$ of methylprednisolone twice a day, and after a rapid clinical response, switched to high dose oral corticosteroids. His dyspnea, wheezing, hypoxia and chest x-ray opacities rapidly resolved. His absolute eosinophil count decreased to 20 eosinophils/yL. He was discharged from the hospital after 5 days with a planned slow taper of his oral prednisone dose.

\section{References}

1. Radiopedia.org. Available at: http://radiopaedia.org/articles/reverse-bats-wingpulmonary-opacities (accessed 10/23/14).

2. King TE Jr. Role of bronchoalveolar lavage in diagnosis of interstitial lung disease. UpToDate. Available at: http://www.uptodate.com/contents/role-of-bronchoalveolarlavage-in-diagnosis-of-interstitial-lung-disease (accessed 10/23/14, requires subscription).

3. Alam M, Burki NK. Chronic eosinophilic pneumonia: a review. South Med J. 2007;100(1):49-53. [CrossRef] [PubMed] 